\title{
Coombs-Negative Haemolytic Anaemia, Direct Hyperbilirubinaemia and Splenomegaly: A Rare Amalgam
}

\author{
Radhika Kulkarni, Amjad Basheer, Aziz Khan \\ UCONN Health, Farmington, CT, USA
}

Received: $12 / 03 / 2021$

Accepted: 19/03/2021

Published: 08/04/2021

\begin{abstract}
How to cite this article: Kulkarni R, Basheer A, Khan A. Coombs-negative haemolitic anaemia, direct hyperbilirubinaemia and splenomegaly: a rare amalgam. EJCRIM 2021;8: doi:10.12890/2021_002477.
\end{abstract}

Conflicts of Interests: The Authors declare that there are no competing interests.

This article is licensed under a Commons Attribution Non-Commercial 4.0 License

\section{ABSTRACT}

Introduction: Epstein-Barr virus (EBV) is notorious for its varied presentation in adults. Reactivation of EBV can occur at any time and is often due to weakened cellular immunity.

Case description: Here we report the case of a young woman with no previous medical history who presented with cholestatic hepatitis, Coombs-negative haemolytic anaemia and splenomegaly. Due to the initial disjointed picture with no other localizing symptoms, she underwent extensive work-up for the same.

Discussion: EBV has been associated with many malignancies, autoimmune diseases and chronic fatigue syndrome. EBV causes elevated liver enzymes; however, cholestatic hepatitis is exceedingly rare, with only a few cases reported. Haemolytic anaemia is a common complication of EBV infection and is often Coombs positive.

Conclusion: EBV testing should be considered before more invasive and expensive work-up in a patient presenting with multi-systemic abnormalities.

\section{LEARNING POINTS}

- Epstein-Barr virus (EBV) can have myriad manifestations in all age groups.

- Coombs-negative haemolytic anaemia can occur as a complication of EBV.

- EBV testing should be considered prior to more expensive work-up in anyone presenting with abnormalities in the reticuloendothelial system.

\section{KEYWORDS}

Herpesvirus 4, direct Coombs test, cholestasis, splenomegaly

\section{INTRODUCTION}

Epstein-Barr virus (EBV), although a common disease of adolescence, is notorious for its unusual manifestations in adults. Its ability to spread through the reticuloendothelial system results in varied clinical presentations. Hepatitis and Coombs-positive haemolytic anaemia are well-known complications of EBV. However, cholestatic hepatitis and Coombs-negative haemolytic anaemia are exceedingly rare and their co-presentation in the same patient has not been previously reported according to our knowledge. 


\section{CASE DESCRIPTION}

A 19-year-old woman with no significant past medical history except for infectious mononucleosis with EBV 18 months previously, presented with a 3-day history of jaundice and high-coloured urine. She had had no symptoms in the preceding days, such as fever, chills, abdominal pain, nausea, vomiting or fatigue, and sought medical help solely after noticing the yellowish discoloration of her eyes and urine. She did not drink alcohol and denied usage of any medications, including herbal medications. She had no significant travel history or previous history of blood transfusions, but had had a tattoo 1 year ago. Family history was negative for anaemia and liver diseases. On examination, she appeared well but had deep icterus of the sclera and skin. She had a mildly tender spleen, palpable $2-3 \mathrm{~cm}$ below the left costal margin. No lymph nodes were palpable, and no skin rash was seen. Laboratory results were notable for Hgb 8.1 g/dl, AST 153 U/I, ALT 145 U/l, ALP 253 $\mathrm{U} / \mathrm{l}$, total bilirubin $11.2 \mathrm{mg} / \mathrm{dl}$, direct bilirubin $8.7 \mathrm{mg} / \mathrm{dl}$, LDH $787 \mathrm{U} / \mathrm{l}$, haptoglobin < $8 \mathrm{~g} / \mathrm{dl}$, and reticulocyte index 21\%. The direct Coombs test and the Monospot test were negative. CT of the abdomen revealed splenomegaly with no evidence of intra or extrahepatic hepatobiliary obstruction. This disjointed picture, along with an initial negative Monospot test, posed a diagnostic dilemma that led to extensive infectious, hepatic and haematological work-up. The patient was tested for tick-borne disease, hepatitis, HIV, CMV and COVID-19. Examination of the peripheral smear revealed no schistocytes; however, numerous bizarre appearing lymphocytes were seen (Fig. 1). As her diagnosis remained a mystery even after 3 days of hospitalization, a work-up for autoimmune hepatitis and Wilson's disease was carried out. Ultimately on day 4 of hospitalization, her EBV IgG and IgM tests were positive for IgG $>750 \mathrm{U} / \mathrm{ml}$, IgM >160 U/ml, which were significantly higher than her titres from the year before (IgG $59.8 \mathrm{U} / \mathrm{ml}$, IgM $36.5 \mathrm{U} / \mathrm{ml}$ ). She spiked a fever and developed pharyngitis on day 4 of hospitalization. Flow cytometry showed the expansion of CD8-positive T-cells. Her EBV PCR showed 23,500 copies/ml. Her haemoglobin remained stable over the next 3 days, and she was discharged home in a stable condition.

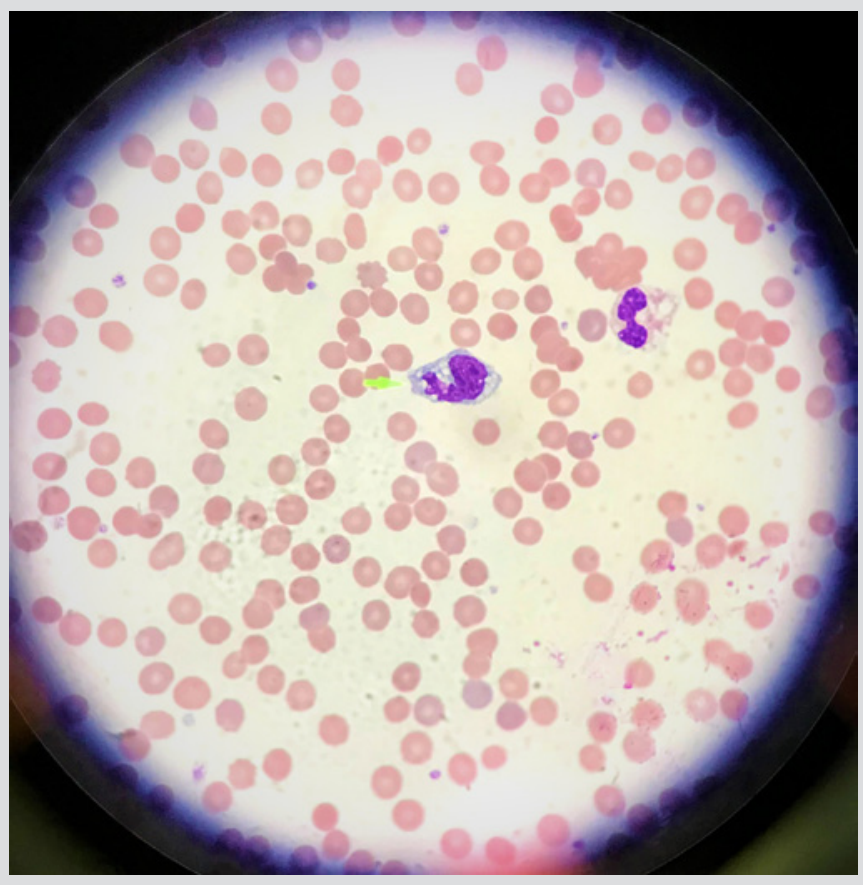

Figure 1. Peripheral smear, 100x: large, atypical lymphocyte (green pointer) with cytoplasmic vacuolation. There is no evidence of schistocytes

\section{DISCUSSION}

Two broad differentials should be considered in a patient presenting with abnormalities of the reticuloendothelial system: infectious and neoplastic. We wish to reiterate Occam's razor - the importance of considering one common aetiology even in the presence of multisystemic dysfunction. EBV is notorious for its varied presentations. EBV causes elevated liver enzymes; however, cholestatic hepatitis is exceedingly rare, with only a few cases reported ${ }^{[1,2]}$. The pathogenesis of cholestasis in EBV is poorly understood. It may be due to host responsemediated inflammation of bile ducts rather than the direct effects of the virus ${ }^{[3]}$. Ultrasound of the abdomen is a cost-effective tool to begin work-up of cholestatic liver injury. Once excluded, medication review and infectious work-up should be pursued next. EBV is known to be associated with cold agglutinin-haemolytic anaemia, which is often Coombs positive. There are rare case reports of Coombs-negative haemolytic anaemia ${ }^{[4]}$. What is the underlying process driving the haemolysis? The pathophysiology is poorly understood. Perhaps the conventional tube technique used to detect the RBC-bound antibody does not pick up low titres of antibody. In such patients, one could try 


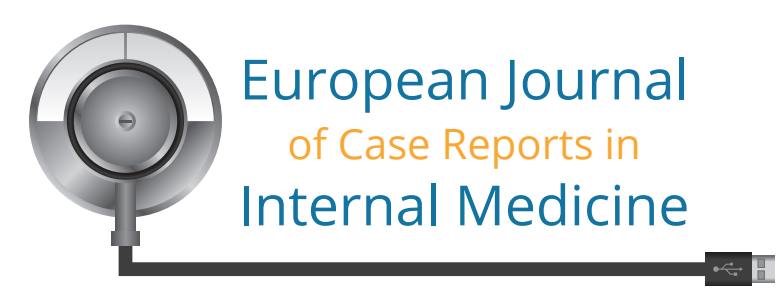

the immunoradiometric assay (IRMA) to identify the RBC-bound IgG (RBC-IgG) ${ }^{[5]}$. Reactivation of EBV can occur at any time and is often due to weakened cellular immunity. It has been associated with many malignancies, autoimmune diseases and chronic fatigue syndrome [6]. Whether reactivation disease may herald the onset of autoimmune disease or lymphoproliferative disorders is unknown. In conclusion, one must always rule out EBV before ordering more lengthy and costly investigations in patients presenting with signs of reticuloendothelial dysfunction.

\section{REFERENCES}

1. Edoute Y, Baruch Y, Lachter J, Furman E, Bassan L, Assy N. Severe cholestatic jaundice induced by Epstein-Barr virus infection in the elderly. J Gastroenterol Hepatol 1998;13(8):821-824.

2. Teijido J, Tillotson K, Liu JM. A rare presentation of Epstein-Barr virus infection. J Emerg Med 2020;58(2):e71-73.

3. Feranchak AP, Tyson RW, Narkewicz MR, Karrer FM, Sokol RJ . Fulminant Epstein-Barr viral hepatitis: orthotopic liver transplantation and review of the literature. Liver Transplant Surg 1998;4(6):469-476.

4. Shaqsi LA, Beamish J. P566 EBV related autoimmune haemolytic anaemia with a negative direct coomb's test - a case report. Archives of Disease in Childhood 2019;104:A380

5. Kamesaki T, Oyamada T, Omine M, Ozawa K, Kajii E. Cut-off value of red-blood-cell-bound IgG for the diagnosis of Coombs-negative autoimmune hemolytic anemia. Am J Hematol 2009;84(2):98-101.

6. Kerr JR. Epstein-Barr virus (EBV) reactivation and therapeutic inhibitors. J Clin Pathol 2019;72(10):651-658. 